\title{
Capacity of Hybrid Cellular-Ad hoc Data Networks
}

\author{
Lap Kong Law, Srikanth V. Krishnamurthy, and Michalis Faloutsos \\ Department of Computer Science \& Engineering \\ University of California, Riverside \\ Email: \{lklaw,krish,michalis\}@cs.ucr.edu
}

\begin{abstract}
In this paper, towards improving spatial reuse in a cellular network, we consider augmenting it with wireless ad hoc connectivity. The coverage area of each base-station is reduced and the users that are within the area relay traffic to nodes outside the area; these users further relay data to more distant users within the cell. The resulting network is referred to as a hybrid network. While this approach can result in shorter range higher-rate links and improved spatial reuse which, together favor a capacity increase, it relies on multi-hop forwarding which is detrimental to the overall capacity. Our objective in this work is to evaluate the impact of these conflicting factors on the capacity of the hybrid network and determine if this capacity is higher than that of the original cellular network. We formally define the capacity of the network as the maximum possible downlink throughput under the conditions of max-min fairness. We analytically compute the capacity of a two-dimensional hybrid network with regular placements of base-stations (BSs) and users. We validate our analytical results via simulations. Our studies demonstrate that capacity improvements are possible in certain parametric regimes in which the penalty due to multi-hop relaying does not outweigh the gains due to spatial reuse and shorter higher-rate links. Our simulations also demonstrate that if the users are placed randomly, the behavioral results are similar to that with regular placements of users.
\end{abstract}

\section{INTRODUCTION}

The capacity of a cellular data network can be improved by creating a larger number of smaller cells, each of which houses an expensive base-station (BS). The benefit of such an approach is the increased spatial reuse of the spectrum. Alternatively, in order to increase spatial reuse, cellular networks may be augmented with ad-hoc wireless connectivity; this is attractive as compared to the former approach in terms of the incurred cost [1], [2]. We call these latter type of networks hybrid cellular-ad hoc networks or simply hybrid networks. A natural question that arises is: do such hybrid networks indeed offer a higher capacity than the original pure cellular network?

While there has been a surge in interest in modelling various kinds of hybrid networks (to be elaborated in section II) [3], [4], [5], [2], [6], [1], [7], [8], [9], [10], [11], the above question has not been answered to date. While the use of shorter range and hence, higher rate wireless ad-hoc links may improve spatial reuse (more simultaneous transmissions can occur) [12], the use of multi-hop relaying increases the number of wireless hops traversed and this reduces the achievable throughput. Given the two conflicting factors, it is unclear whether or not the capacity of the network will in fact increase relative to the original pure cellular network. In this paper, we determine under what conditions and by how much is the downlink capacity of a hybrid cellular-ad hoc network higher or lower than that of the original pure cellular network.

The hybrid network in brief: To describe the hybrid network in more detail, consider a two-dimensional hexagonal

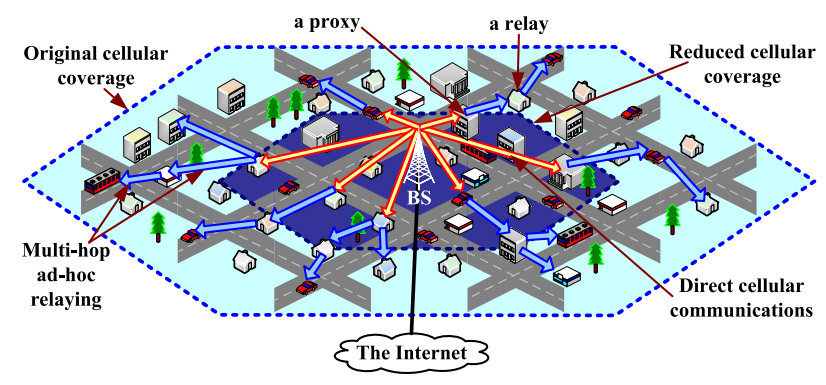

Fig. 1. A pictorial representation of a hybrid network.

hybrid network as shown in Fig. 1. In this network, only users that are within the reduced cellular coverage (dark hexagon) receive downlink traffic directly from the BS; this direct link between the BS and such users is called the infrastructure component of the hybrid network. The reduced cellular coverage enhances the transmission efficiency of the BS since the directly connected users, being close to the BS, usually have good channels to the BS; thus, the BS can now support higher rates to these users. On the other hand, users that are outside the reduced cellular coverage require that the directly connected users act as proxies and forward traffic from the BS. In fact, only a subset of these outside users may directly receive traffic from the proxies. These users will then have to act as relays and forward traffic to other users that are further away from the BS. The part of the network that delivers the data from the proxies to the outside users, is called the ad hoc component of the hybrid network.

The Capacity of the Hybrid Network: We define the capacity of the hybrid network to be the maximum possible network throughput under the conditions of max-min fairness ${ }^{1}$ [13]. We compute a closed form expression for the capacity of the hybrid network; the capacity varies with system parameters as we articulate later and this defines a region. Note that for a defined set of parameter values the computed capacity may or may not be achievable in practice; however the achievable throughput will always be less than or equal to the capacity. The computed expression can also be trivially used to compute the capacity of the original pure cellular network.

The distinguishing aspects of our work can be summarized as follows:

- In computing the downlink capacity, we consider interference from surrounding cells. We account for such interference when modelling both direct BS-to-user communications and ad hoc user-to-user communications.

- Unlike in previous efforts (such as [7], [11]), our model

${ }^{1}$ We seek to avoid the starvation of users with poor channel conditions i.e., consider a scenario in which a fair share of the network capacity is provided to to each user. 
reflects the impact of the distance spanned by the link on the achieved rate. In other words, we examine the impact of rate versus range tradeoffs on the capacity of the hybrid network.

- Unlike most previous efforts, our model accounts for the fact that the BS is always at one end of every communication. Given that cellular users typically download content from the Internet [14] (as opposed to uploading) our work focuses on downlink communications; uplink communications will be considered in the future. Note that the considered traffic pattern has a big impact on the computed capacity since it affects the extent of possible spatial reuse in the ad-hoc part of the network. With the considered traffic pattern, in the hybrid network, nodes that are closer to the BS carry more traffic and thus, are likely to be active more often. We take this into account in our analysis.

- We consider a fixed spectral band that is shared between the direct cellular communications and the ad hoc communications. Most previous efforts assume the use of an additional spectral band for supporting multi-hop relay communications [4], [2]. Our approach facilitates a fair comparison between the capacities of the hybrid network and its original pure cellular counterpart.

- In addition to validating our analysis, our simulations show that the capacity achieved by placing users randomly within the network is similar to that with the regular placements of users (the analysis assumes regular placements).

Our results in brief: Our analysis computes the downlink capacity in hybrid networks. We compare this capacity with that of the original pure cellular network. The pure cellular network is simply a specific case of the hybrid network when no ad-hoc component exists. Thus the capacity of the pure cellular network is trivially computed from the same analysis and with the same set of assumptions; this makes the comparison meaningful. Our studies result in the high level conclusion that the hybrid network can indeed offer capacity benefits as compared to the original pure cellular network but only when three key parameters discussed below, are appropriately tuned. In the best case, the capacity of the hybrid network can be about $70 \%$ higher than that of the corresponding original pure cellular network.

Fundamental Trade-offs: We find that the capacity of the hybrid network depends on the following parameters: 1) the size of the BS footprint, 2) the spectrum allocation between the BS-to-user links and the user-to-user links, and 3) the transmission range of the multi-hop ad-hoc wireless links. The first parameter $b$, the size of the BS footprint, determines the region of direct cellular coverage (as described earlier). The smaller the footprint, the higher will be the rate at which the BS can transmit to its directly connected users; however this will lead to a higher multi-hop forwarding overhead. Second, the fixed spectral bandwidth has to be appropriately apportioned between the infrastructure and the ad-hoc components in order to ensure that one component does not become a bottleneck. The capacity depends on what fraction this spectral bandwidth is assigned to the two components. Finally, we can vary the transmission range $r$, of the ad-hoc links. Choosing a longer range would result in fewer hops; however, the rate on each hop will be low. One could instead choose to use a large number of high-rate, short-range links at the cost of traversing more hops.

Roadmap: The rest of the paper is organized as follows: In the next section, we discuss related work. In section III, we formulate the problem. In section IV, we compute the downlink capacity of the hybrid network. A description of our simulation experiments and our performance evaluations form section V. We conclude in section VI.

\section{RELATED WORK}

Most previous efforts on computing the capacity of wireless networks either consider a pure ad-hoc or a cellular network [15], [16]. There are a limited number of efforts on various kinds of hybrid networks. The distinguishing aspects of our work compared to these efforts were highlighted in Section I.

We discuss the previous efforts in brief below and enunciate on how our work differs from these efforts in specific aspects.

Capacity of hybrid wireless networks: In [1], a hybrid network with a sparse layout of base stations connected by high-bandwidth links, is studied. It is shown that with $n$ ad-hoc nodes and $m$ base stations, the benefits provided due to the base stations are insignificant when $m$ grows asymptotically slower than $\sqrt{n}$. In [8], in a similar network setting, it is shown that $\Theta(1)$ per node throughput can be achieved when the nodes can optimally control their transmission powers. In [9], it is shown that a hybrid network with $n$ wireless nodes and $n^{d}$ access points, inter-connected by wires, can achieve throughput gains only when $\frac{1}{2}<d<1$. Unlike our work, the above efforts assume that the traffic flows are established between randomly chosen ad-hoc nodes and the base stations or access points are for relaying purposes only.

In [7], [11], the authors consider a hybrid network that is similar to what we consider, but with a small number of relays and a small number of hops. However, they do not consider rate variability with range; furthermore, the transmission range of relays is always equal to the distance between the relays. Furthermore, they do not consider load heterogeneity of relays due to the downlink traffic flow pattern. [7] provides a simple informal analysis on the capacity gain and evaluates the throughput gain of the network with 4 relays. The work in [11] evaluates the throughput gain of the network by considering geographical routing. In [10], the authors evaluate the trade-offs between channel reuse efficiency and increased interference in simple and small hybrid network topologies.

Hybrid network architectures: In [3], a Multihop Cellular Network (MCN) is proposed. It is shown that the throughput with the MCN exceeds that of the pure cellular network. However, it is assumed that the transmission range of the BSs is the same as that of the mobile stations (MSs). BSs are for relaying purpose only. In [4], an integrated cellular and ad-hoc relaying system (iCAR) is proposed; ad-hoc relaying stations (ARS) are strategically deployed to improve call service quality; capacity gain is not the main focus of this work. Our work differs from the above efforts in that the transmission range of a BS (the size of the BS footprint) can be varied and is independent to the transmission range of the MSs. Furthermore, these efforts assume peer-to-peer traffic patterns and do not consider rate variability with range. 


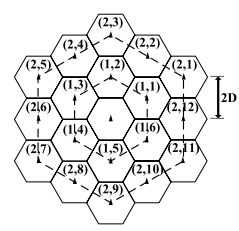

(a)

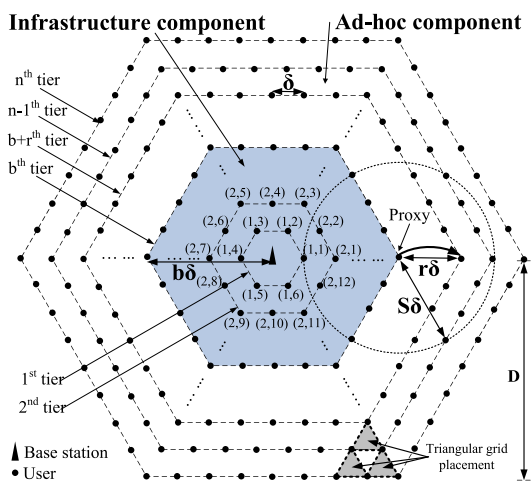

(b)
Fig. 2. Hexagonal hybrid network. (a) Cellular arrangement (cells are arranged in levels). (b) Users arrangement within a cell (Users are arranged in tiers)

In [5], the authors consider the ad-hoc network model for wireless packet data networks and show that this can support better spatial reuse and result in better throughput per unit power spent. However, again, the BSs are for relaying purposes only. In [2], the authors propose a unified cellular and adhoc network (UCAN) architecture to improve individual user throughput and the aggregate downlink throughput. However, UCAN assumes the use of an additional spectral band for adhoc communications.

\section{Problem Formulation}

In this section we describe the network being considered and the models that we use; we list and justify the assumptions that we make. We re-postulate some of the definitions from Section I for completeness.

The Hybrid Network: The hybrid network consists of two components: 1) the infrastructure component and 2) the adhoc component. The infrastructure component refers to the part of network within which, a user can communicate with its serving BS directly. The ad-hoc component refers to the part of the network from which a user cannot communicate (effectively) with its serving BS directly; it requires its neighbors to relay traffic from the BS (across multiple hops). For clarity and the purposes of analysis, we use the concept of a base station footprint (BS footprint). We formally define it to be the maximum distance from the BS within which a given user can communicate directly with the BS given a data rate requirement.

The region of interest (see Fig. 2(a)) is organized into hexagonal cells and each cell contains a single BS located at the center of the cell. The distance between any two BSs is equal to $2 D$, as shown in Fig. 2(a). For each BS, the surrounding BSs are arranged in levels and indexed as shown in Fig. 2(a) (dotted concentric hexagons). We assume that a direct transmission from a BS to a user is subject to interference from infinite levels of surrounding BSs. We limit ourselves to downlink transmissions i.e., transmissions from the BS to the users, since this is likely to represent the biggest portion of traffic flow given that Internet traffic largely consists of web downloads.

In order to make our analysis tractable, we assume that users are uniformly and regularly placed in the network. We relax this assumption in our simulations and consider the more complex case where the users are randomly placed. We consider the triangular grid placement of users (see Fig. 2(b)) since with this arrangement, users can be placed within a hexagonal cell perfectly with the highest density, for any given separation between adjacent users. In our formulation, the users are arranged and indexed in tiers; the tiers are represented by the dotted concentric hexagons in Fig. 2(b).

Configuration and Spectral Allocation: We assume that all users are equipped with two communication interfaces: 1) the cellular interface, and 2) the ad-hoc interface. A user within the BS's footprint, uses its cellular interface to communicate with the BS; it uses its other interface to communicate with the users outside the BS's footprint. Note here that the two interfaces operate on non-overlapping channels. The channels are half-duplex. A fixed spectral bandwidth is shared between the two components. Let us denote by $B$, the total available bandwidth (in $\mathrm{Hz}$ ); let $\gamma B$ (where $0<\gamma \leq 1$ ) be the fraction of the total bandwidth that is allocated for infrastructure communications. Then, the bandwidth allocated for multi-hop ad-hoc communications would be $(1-\gamma) B$.

Channel Properties and Models: We consider a simple path-loss channel propagation model. In particular, $P_{R X}=$ $\frac{P_{T X}}{d^{\alpha}}$ where, $P_{T X}$ is the average transmission power, $P_{R X}$ is the average received signal power, $d$ is the distance between the transmitter and the receiver, and $\alpha$ is the path loss exponent; usually $\alpha \geq 2$. With a goal of capturing long term channel effects, multipath fading is not considered. We also do not consider shadow fading; this will be considered in future work.

To compute the capacity of a wireless channel, we model the co-channel interference as Gaussian ${ }^{2}$ as in [17], [12]; then the "channel capacity" is given by Shannon's capacity formula [18]:

$$
\text { Capacity }=W \log _{2}(1+S I N R)
$$

where $W$ is the bandwidth (in $\mathrm{Hz}$ ), and $S I N R$ is the Signal-toInterference-plus-Noise Ratio. Note that the channel capacity is the maximum achievable throughput on a link and may not be achieved in practice due to protocol semantics and overheads.

Transferring traffic from a BS to the users within its footprint: As mentioned earlier, a BS transmits directly only to the users within its footprint. BSs perform transmissions in Time Division Multiplexed (TDM) mode, i.e., at any given point in time, a BS transmits to only one user, and at full power, $P_{B S}$. The transmission power $P_{B S}$, is the same for all BSs in the network. We consider an aggressive approach in which all the BSs are transmitting all the time. Therefore, a receiving user experiences interference from all other $\mathrm{BSs}^{3}$. This model has been proven to maximize the overall throughput of the system [19] and has been employed as the transmission strategy for 1xEV-DO [20].

An infrastructure link can be formed between a user and its closest BS. We define the infrastructure link capacity (ILC) as the maximum transmission rate that can be achieved if the user is the only user that is served by the BS. With our model, the ILC of a user is dependent on its location

\footnotetext{
${ }^{2}$ In the presence of a large number of independent interferers, the Central Limit Theorem supports this assumption.

${ }^{3}$ Such an aggressive approach would typically increase the collisions at users at the boundary of the cell in a pure cellular network. However, such users will not typically communicate with the BS directly in the hybrid network due to the smaller BS footprint. These users instead use multi-hop ad-hoc links for communications and thus, the above effects are less pronounced.
} 


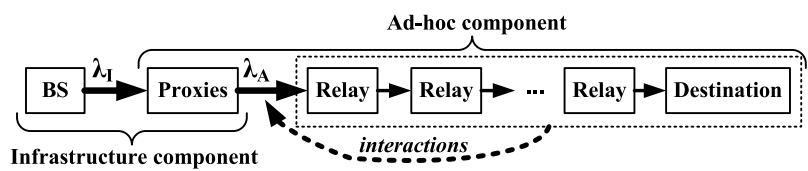

Fig. 3. An abstract representation of a hybrid network.

only. The SINR of a user and thus the ILC can be easily computed by Equation (1). However, the actual serving rate of a user depends on the number of users, their ILC's and the transmission scheduling strategy of the BS. To determine the transmission strategy of BSs, cellular network providers usually consider both throughput and fairness issues [21]. Here, we consider the transmission strategy that achieves maximum fairness.

Traffic flow to the ad-hoc component via the proxies and the relays: Users that lie outside the BS footprint will have to rely on their neighbors to act as relays to deliver traffic from the BS. Note that these relays may in turn depend other neighbor relays. Ultimately, the relays that are within the BS footprint relay traffic to those users that are outside the footprint (using the ad-hoc interface). Thus, these users act as proxies to the BS.

As in typical ad-hoc networks, carrier sensing is employed [17] within the ad-hoc component. All users use the same transmission power, $P_{\text {user. }}$ A user can transmit (using its adhoc interface) only if there is no other active transmitter that is located within its sensing range [12]. Note that the sensing range is only a function of the transmission power and the sensitivity of receivers $[22]^{4}$.

The capacity of an ad-hoc link depends on the distance between the transmitting and receiving user. In order to sustain a desired transmission rate on an ad-hoc link, the maximum distance that a transmission can span must be restricted. This distance is the transmission range (or simply range) of the link. Choosing a shorter range increases the achievable rate on each link; however, it also increases the number of hops needed to reach a destination user. We examine the trade-offs between the two effects ${ }^{5}$.

We assume that a relay path from a proxy to a destination user, is the minimum-hop path, given the transmission range. This assumption is commonly made in previous capacity analyses [15], [23] since it minimizes the relaying overhead incurred in the network.

Impact of Density: Note here that the imposed span of the links in the network and therefore the achievable rate is dictated by the density of users in the network. If the network is sparse, one would be forced to establish longer links and therefore the achievable rates on the links will be limited. If on the other hand, there is a dense population of ad hoc users, one could potentially form shorter and therefore higher-rate links. We study the impact of user density in our performance evaluations. Note however that we do not consider extremely sparse cases where ad hoc links can only support extremely low data rates since these cases are likely to be of no interest.

\footnotetext{
${ }^{4}$ In IEEE802.11a, a typical transmission power is $15 \mathrm{dBm}(32 \mathrm{~mW})$ and the receiver sensitivity is around $-90 \mathrm{dBm}\left(1 e^{-9} \mathrm{~mW}\right)$. With this, the carrier sensing range $S \approx 421 \mathrm{~m}$.

${ }^{5}$ This distinguishes our work from other similar studies wherein, the variability in the achievable rate with distance is not considered.
}

Metrics of Interest: We compute the capacity when all users receive approximately the same throughput from the BS (max-min fairness). This avoids starvation for users with poor channel conditions. Thus, the capacity is the maximum possible uniform throughput under the consideration of maxmin fairness. We determine the maximum possible uniform throughput of: (a) the infrastructure component, and (b) the ad-hoc component, separately. Depending upon the key system parameters (discussed in Section I) the capacity bottleneck could appear either in the infrastructure part or the ad-hoc part. Therefore, the maximum possible uniform throughput of the hybrid network is the minimum of the maximum possible uniform throughputs of the two components. Fig. 3 depicts an abstract representation of a hybrid network. The capacity between the BS and the proxies (as shown) is the uniform throughput of the infrastructure component, $\lambda_{I}$. The proxies deliver information to the rest of the users in the adhoc component at a rate we call the uniform throughput of the ad-hoc component, $\lambda_{A}$. We wish to point out here that proxy-to-relay communications and relay-to-relay (and relayto-destination) communications share the available bandwidth and this will impact the maximum achievable $\lambda_{A}$. The uniform throughput of the hybrid network $\lambda_{H}$, is then,

$$
\lambda_{H}=\min \left(\lambda_{I}, \lambda_{A}\right)
$$

\section{Computing the Downlink Capacity}

In this section, we analytically compute the capacity of the hybrid network.

As discussed in section III, we arrange users in tiers within a cell. A tier essentially consists of the users that lie on a hexagon of a certain major radius. Let $n$ be the number of tiers of users in a cell and let $\delta$ be the distance between adjacent users. Then, the radius of a cell is $D=n \delta$. Note here that $n$ and $\delta$ together determine the density of users in the network. Let $r$ denote the transmission range of an ad-hoc link (for a given transmission rate requirement, as discussed in section III), $S$ the sensing range of each user, and, $b$ the size of the BS footprint. Note that $S \geq r$ and $n \geq b \geq r^{6}$. The above distances are defined relative to the distance between users i.e., in terms of units each of which is of length $\delta$. A user in a cell is indexed $U S E R_{i, j}$, where $i(i \geq 1)$ represents the tier to which the user belongs, and $j(1 \leq j \leq 6 i)$ identifies the user within that tier (see Fig. 2(b)). Given the minimum-hop routing strategy, each user, when establishing an ad-hoc link, selects the neighbor that is furthest from itself, from among its reachable neighbors (those that fulfil the transmission rate requirement) in order to minimize the number of relay operations (we call this the relaying overhead). With this, users in the $i^{\text {th }}$ tier will always attempt to form an ad-hoc link with users in the $(i-r)^{t h}$ and $(i+r)^{t h}$ tiers, if these tiers exist. Furthermore, users that are located between the $(b-r+1)^{t h}$ and the $b^{t h}$ tiers are potential proxies.

Uniform Throughput of the Infrastructure Component: We wish to maximize $\lambda_{I}$ such that all users receive the same throughput (max-min fairness). We assume that the ILCs of users that are located within the same tier are the same and

\footnotetext{
${ }^{6}$ We only consider the case where $b \geq r$ since the direct link from the more powerful BS is likely to be at least as long as an ad-hoc link.
} 
equal to the best ILC possible for any user within the tier ${ }^{7}$. Let $c_{i}$ denote the ILC of a user in the $i^{t h}$ tier and $\tau_{i}$ the fraction of time that the BS transmits to a user in the $i^{\text {th }}$ tier. The maximum value of $\lambda_{I}$ is computed by solving the following:

$$
\begin{array}{lll}
\operatorname{maximize} & \lambda_{I} & \forall i=1, . .,(b-r), \\
\text { subject to } & \tau_{i} c_{i}=\lambda_{I} & \\
\tau_{j} c_{j}= & \left(1+\sum_{m=1}^{\left\lfloor\frac{n-j}{r}\right\rfloor} \frac{6(j+m r)}{6 j}\right) \lambda_{I} & \forall j=(b-r+1), . ., b, \\
& \sum_{k=1}^{b} 6 k \tau_{k}=1 &
\end{array}
$$

The first constraint mandates that the throughput of the nonproxy users in the infrastructure component is the same and is equal to $\lambda_{I}$. The second constraint requires that each proxy receives a throughput of $\lambda_{I}$ for itself and obtains an additional throughput of $\left(\sum_{m=1}^{\left\lfloor\frac{n-j}{r}\right\rfloor} \frac{6(j+m r)}{6 j}\right) \lambda_{I}$ for the users in the adhoc component, for which it acts as a proxy. Note that $\sum_{m=1}^{\left\lfloor\frac{n-j}{r}\right\rfloor} \frac{6(j+m r)}{6 j}$ is the average number of users in the adhoc component that are served by a proxy in the $j^{\text {th }}$ tier. The third constraint ensures that the sum of the fractional shares assigned to the users within the infrastructure component does not exceed unity. When $c_{i} \mathrm{~s}, \forall i=1, \ldots, b$ are determined, the maximization problem (3) can be solved as a linear program.

To determine $c_{i}$ s, we compute the $S I N R$ of a user in the $i^{t h}$ tier of users. We denote by $d_{i, j}$ the distance between $U S E R_{i, j}$ and its serving BS. $D_{i, j}^{u, v}$ is the distance between $U S E R_{i, j}$ and the surrounding base station $\mathrm{BS}_{u, v}{ }^{8}$. Then, the $S I N R$ of $U S E R_{i, j}$ (and an upper bound on the SINR of the users in the $i^{\text {th }}$ tier) is given by:

$$
\begin{array}{ll}
\text { SINR } R_{U S E R_{i, j}} & \frac{\frac{P_{B S}}{\left(d_{i, j}\right)^{\alpha}}}{\sum_{u=1}^{\infty} \sum_{v=1}^{6 u}\left[\frac{P_{B S}}{\left(D_{i, j}^{u, v}\right)^{\alpha}}\right]} \\
\leq & \left.\frac{\left(\frac{2}{\sqrt{3} i \delta}\right)^{\alpha}}{\sum_{u=1}^{\infty} \sum_{v=1}^{6 u}\left[\frac{1}{\left(D_{i, j}^{u, v}\right)^{\alpha}}\right]} \text { (since } d_{i, j} \geq \frac{\sqrt{3} i \delta}{2}\right) \\
< & \frac{\left(\frac{2}{\sqrt{3} i \delta}\right)^{\alpha}}{\sum_{u=1}^{\infty} \frac{6 u}{(2 u D)^{\alpha}}}=\frac{2^{2 \alpha-1} n^{\alpha}}{\sqrt{3}^{(\alpha+2)} i^{\alpha} \zeta(\alpha-1)}
\end{array}
$$

where, $D=n \delta$ and $\zeta(\alpha)$ is the Riemann zeta function representing $\sum_{x=1}^{\infty} \frac{1}{x^{\alpha}}$. Noise is ignored since we consider an interference dominated environment. In the last step of the computation, we take advantage of the fact that the interference experienced by $U S E R_{i, j}$ from any particular level of surrounding BSs must be greater than or equal to the interference experienced if the $U S E R_{i, j}$ was located at the center of the serving cell; we also take advantage of the fact that the distance between the center of the serving BS and any BS in the $u^{\text {th }}$ level of surrounding BSs is at most $2 u D$. Thus, $\sum_{v=1}^{6 u}\left[\frac{1}{\left(D_{i, j}^{u, v}\right)^{\alpha}}\right]>\frac{6 u}{(2 u D)^{\alpha}}$.

With the above, the ILC, $c_{i}$, is computed using Equation (1) to be:

$$
c_{i}=\gamma B \times \log _{2}\left(1+\frac{2^{2 \alpha-1} n^{\alpha}}{\sqrt{3}^{(\alpha+2)} i^{\alpha} \zeta(\alpha-1)}\right)
$$

\footnotetext{
${ }^{7}$ This provides an upper bound on the achievable throughput.

${ }^{8} \mathrm{BS}_{u, v}$ is the BS that is located in the $u^{t h}$ level of surrounding BSs and with index $v$ in that level. Note that $u$ and $v$ are defined with respect to the current cell where user ${ }_{i, j}$ is located. Refer to Fig. 2 for the naming convention of BSs and users.
}

Then, $\lambda_{I}$, is found by solving the maximization problem (3) by using standard linear programming techniques.

Uniform Throughput of the Ad-hoc Component: Next, we compute the maximum possible value for $\lambda_{A}$. Given that we only consider downlink traffic, the traffic from the BS must flow through the proxies to enter the ad-hoc component. Therefore, the uniform throughput in the ad-hoc component depends on how well the proxies transport traffic. To this end, we define and compute the following two quantities: (a) the Normalized Relay Load (NRL): The total relay load (due to proxy and relay transmissions) incurred for each unit of traffic delivered per user in the ad-hoc component, and (b) the One Hop Throughput (OHT): The maximum total one-hop throughput (due to proxy and relay transmissions) in the adhoc component. Since the uniform throughput of the ad-hoc component is $\lambda_{A}$, the total traffic load that must be generated in the ad-hoc component is at least NRL $\times \lambda_{A}$. This value cannot exceed and in the best case, can only be equal to the maximum one-hop throughput in the ad-hoc component, i.e.,

$$
\mathrm{NRL} \times \lambda_{A} \leq \mathrm{OHT}
$$

Given the above inequality, if we are able to compute OHT and NRL, we can compute an upper bound on $\lambda_{A}$.

Computing NRL. We first compute NRL. As the distance between the destination user and the BS increases or the transmission range of an ad-hoc link decreases, the number of relay operations (proxy and relay transmissions) needed and thus, the relay load generated increases. As per the minimumhop routing strategy, the relay load that is incurred by relaying one unit of traffic to a user, say, in the $k^{t h}$ tier (where $k>b$ ), is $\lceil(k-b) / r\rceil$. Since there are $6 k$ users in the $k^{t h}$ tier, the NRL is computed to be:

$$
\mathrm{NRL}=\sum_{k=b+1}^{n} 6 k \times\left\lceil\frac{k-b}{r}\right\rceil
$$

Therefore, the total relay load with a uniform throughput of $\lambda_{A}$, is at least $\sum_{k=b+1}^{n} 6 k \times\left\lceil\frac{k-b}{r}\right\rceil \times \lambda_{A}$.

Computing OHT. To determine OHT, we need to calculate: (a) the transmission rate that can be sustained on an adhoc link given the transmission range and (b) the upper bound on the number of ad-hoc transmissions that can occur simultaneously. The product of the two gives the maximum achievable one-hop throughput in the ad-hoc component. Note that OHT is different from the aggregate one-hop throughput that is defined in [15], [23]. The definition used in [15], [23] assumes that a node always has data to transmit when given the opportunity to transmit and thus, the spatial reuse is maximally exploited. However, as was discussed earlier, due to the direction of traffic flow in the hybrid network, users that are in the vicinity of the BS have to transmit more often than the others that are not directly connected to the BS. A user may not have data to transmit when given the opportunity to do so. We will take this heterogeneous load distribution into account when computing OHT.

We first compute the transmission range of an ad-hoc link

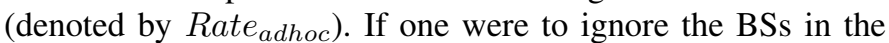
hybrid network, due to the regular placement of users, one would observe an infinite hexagonal grid of users. Let us focus on a specific user (say User A) as shown in Fig. 4. If User A 


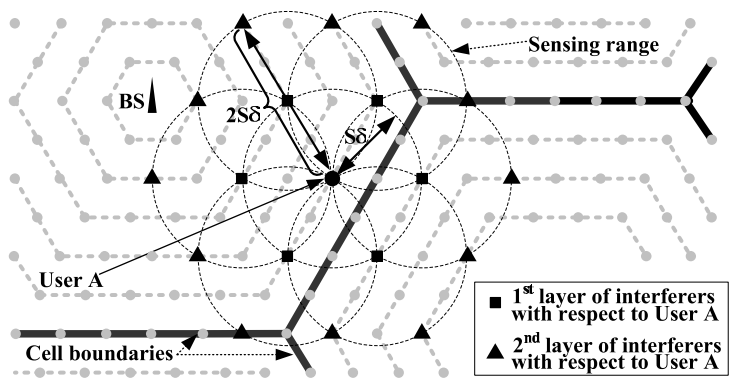

Fig. 4. Layers of interferers with respect to user A.

is an active transmitter, due to the carrier sensing constraint, other simultaneously active transmitters must be outside User A's sensing range i.e., at a distance $\geq S \delta$ from User A. From geometric considerations, it is easy to verify that there can be at most 6 other active interferers that are at a distance of $S \delta$ from User A [24], [17], [12]; these form the $1^{\text {st }}$ layer of interferers with respect to User A (Fig. 4). If these 6 "interferers" are transmitting, it is easy to see that there can be at most 12 other "interferers" that are at a distance of at most $2 S \delta$ from User A, forming the $2^{\text {nd }}$ layer of interferers (Fig. 4), and so on. Therefore, the $t^{t h}$ layer of interferers has $6 t$ interferers that are at a distance of at most $t S \delta$ from User A. Thus, with the maximum number of simultaneously active ad-hoc transmissions, the $S I N R$ at any receiver is computed to be:

$$
S I N R<\frac{\frac{P_{u s e r}}{(r \delta)^{\alpha}}}{\sum_{t=1}^{\infty} 6 t \frac{P_{u s e r}}{(t S \delta)^{\alpha}}}=\frac{S^{\alpha}}{6 r^{\alpha} \zeta(\alpha-1)}
$$

With this, an upper bound on the transmission rate that can be reliably sustained on an ad-hoc link is:

$$
\text { Rate }_{\text {adhoc }}=(1-\gamma) B \times \log _{2}\left(1+\frac{S^{\alpha}}{6 r^{\alpha} \zeta(\alpha-1)}\right)
$$

The second step towards computing OHT is to compute the maximum number of simultaneous ad-hoc transmissions. First, we denote by ProXYTX and RELAYTX, the upper bounds on the number of simultaneous proxy and relay transmissions in the network, respectively (assuming maximum fairness). Then, the upper bound on the number of simultaneous ad-hoc transmissions in the network will be (PROXYTX+RELAYTX). In fact, there is a correlation between PROXYTX and RELAYTX. One may envision that when a proxy transmits a packet, the packet is relayed a number of times (depending on the distance between the proxy and the destination) until it reaches the destination. Given the regular placement of users in our model, we can find an upper bound on RELAYTX in terms of PROXYTX, within a multiplicative factor. We denote by RPRATIO, the above mentioned multiplicative factor. RPRATIO represents the maximum possible ratio of the number of relay transmissions to the number of proxy transmissions in order for every user in the ad-hoc component to receive a unit of data traffic. RPRATIO is thus given by:

$$
\text { RPRATIO }=\frac{\sum_{k=b+r+1}^{n} 6 k\left\lceil\frac{k-(b+r)}{r}\right\rceil}{\sum_{m=b+1}^{n} 6 m}
$$

where, the numerator reflects the number of relay transmissions and the denominator, the number of proxy transmissions. Then the maximum number of actual simultaneous ad-hoc transmissions is upper bounded by PROXYTX $\times(1+$ RPRATIO $)$. Our target is then to compute PROXYTX.

Due to carrier sensing, the fraction of time that a proxy can transmit is affected by the number of proxies and relays that are located within its sensing range and their carried loads (the carried load of a user is the amount of traffic that is to be transmitted by the user). We define the normalized carried load of a user in the $k^{\text {th }}$ tier, $l_{k}$, to be the carried load of a user in the $k^{\text {th }}$ tier such that each user that relies on the considered user as a relay, receives a unit of data traffic. Then $l_{k}$ is given by:

$$
l_{k}=\left\{\begin{array}{cl}
\sum_{m=1}^{\left\lfloor\frac{n-k}{r}\right\rfloor} \frac{6(k+m r)}{6 k} & \text { if } b-r+1 \leq k \leq n-r \\
0 & \text { otherwise }
\end{array}\right.
$$

Essentially, $l_{k}$ is equal to the number of more distant users to which, a user in the $k^{\text {th }}$ tier will relay traffic. Note that when a proxy is forbidden to transmit due to the carrier sensing constraint, there could be at most six other active users in its sensing range. In the best case, the six active users simultaneously transmit while restricting the transmission opportunity of the proxy. This effect causes the fraction of time for which the proxy can transmit to be higher than if the six active users were to transmit sequentially. Therefore, in the best case, the fraction of time that an arbitrary proxy can transmit is the ratio of the proxy's normalized carried load to the sum of its normalized carried load and one-sixth of the normalized carried loads of all other users within its sensing range. However, the determination of the number of interferers in each tier of users within a proxy's sensing range is difficult since it depends on the position of proxy, the size of the sensing range and the orientation of the interfering tiers. We seek to solve this problem by geometry. Fig. 5(a) depicts a cell with hexagons representing the tiers of users. The sensing region of a proxy is shown by the shaded circle (sensing circle). The highlighted line segments inside the circle capture the locations of the interferers. Due to the regular placement of users, if we are able to find the length of each of these line segments, the number of interferers in each tier of interest can be computed. Let us now focus on one particular tier of interferers (say, the $t^{\text {th }}$ tier) and a proxy in the $p^{t h}$ tier. We depict this scenario in Fig. 5(b). In the figure, the hexagon in bold represents the $t^{\text {th }}$ tier of interferers and the circle in bold represents the sensing region of a proxy located in the $p^{t h}$ tier. Then, the interferers of the proxy are located on the highlighted hexagonal line segments that are covered by the proxy's sensing region. Our target is to compute a lower bound on the length of the line segments. Note that $t$ could be either smaller than, larger than or equal to $p$. We draw two dotted circles around the hexagon representing the $t^{\text {th }}$ tier as shown in Fig. 5(b). The inner circle is the largest circle that is contained in the hexagon. The outer circle is the smallest circle that contains the hexagon. We consider two angles: $\theta_{\text {inner }}$ and $\theta_{\text {outer }} . \theta_{\text {inner }}$ is the angle between the lines joining the BS and the intersection points of the inner circle and the sensing circle. Similarly, $\theta_{\text {outer }}$ is the angle between the lines joining the BS and the intersection points of the outer circle and the sensing 


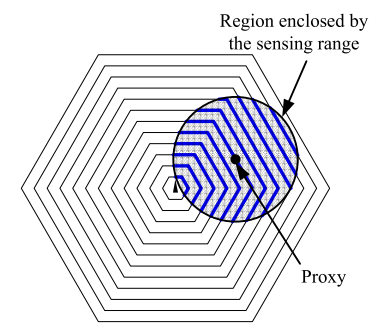

(a)

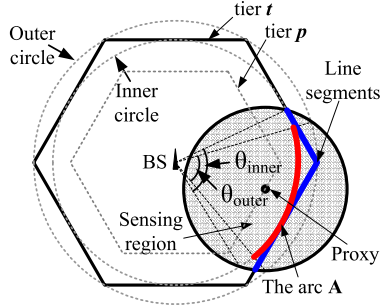

(b)
Fig. 5. Determining the number of interfering users.

circle. The angles are computed using the law of cosines:

$$
\begin{gathered}
\theta_{\text {inner }}=2 \times \cos ^{-1}\left(\frac{\left(\frac{t \sqrt{3}}{2}\right)^{2}+p^{2}-S^{2}}{2 \times \frac{t \sqrt{3}}{2} \times p}\right) \\
\theta_{\text {outer }}=2 \times \cos ^{-1}\left(\frac{t^{2}+p^{2}-S^{2}}{2 \times t \times p}\right)
\end{gathered}
$$

Since the line segments of interest must lie between the inner and the outer circles, the sum of their lengths must be greater than or equal to the length of the arc A (refer Fig. 5); this is of length $\frac{t \sqrt{3}}{2} \times \min \left(\theta_{\text {inner }}, \theta_{\text {outer }}\right)$. Recall that users are regularly placed, adjacent users are of unit distance (unit in $\delta$ ) apart from each other. Therefore, given a proxy in the $p^{\text {th }}$ tier, the lower bound of the number of users from the $t^{t h}$ tier that interferes with its transmission, $I_{p}^{t}$, is simply computed to be:

$$
I_{p}^{t}=\left\lfloor\frac{t \sqrt{3}}{2} \times \min \left(\theta_{\text {inner }}, \theta_{\text {outer }}\right)\right\rfloor
$$

Note that the sensing circle might not intersect with both the inner and the outer circle for some tiers and either one or both angles could be undefined. Since, in this case, the lengths of the line segments that are covered by the sensing circle are very small in general, we can simply ignore the interferers; this is a conservative lower bound on the number of interferers. With this, PROXYTX, the sum of the fraction of transmission times of all proxies is computed as follows:

$$
\text { PROXYTX }=\sum_{p=b-r+1}^{\min (b, n-r)} 6 p \times \frac{l_{p}}{l_{p}+\frac{1}{6}\left[\left(\sum_{t=1}^{n} I_{p}^{t} \times l_{t}\right)-l_{p}\right]}
$$

In what follows, OHT is computed to be:

$$
\text { OHT }=\text { PROXYTX } \times(1+\text { RPRATIO }) \times \text { Rate }_{\text {adhoc }}
$$

By using Equations (6), (7) and (16), the uniform throughput of the ad-hoc component, $\lambda_{A}$, is thus:

$$
\lambda_{A} \leq \frac{\text { PROXYTX } \times(1+\text { RPRATIO }) \times \text { Rate }_{\text {adhoc }}}{\text { NRL }}
$$

We defer a discussion on numerical estimates of the capacity and the interpretations thereof to the next section.

\section{EVALUATIONS}

In this section, we present numerical results derived from our analytical models. In addition, we perform simulations using the ns-2 [25] simulator to validate our analysis. In our simulations, we consider both regular and random placements of users. The results in both cases conform with our analytical results; this suggests that our analytical results are representative of the capacity of hybrid networks.

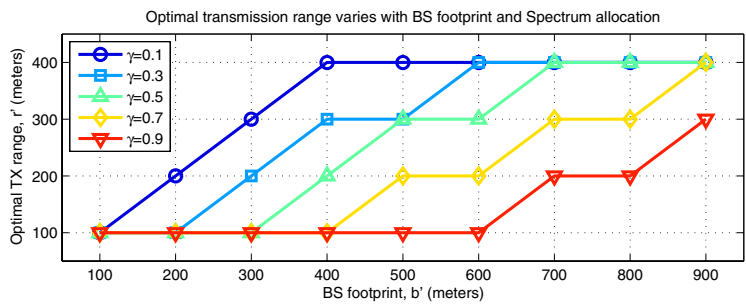

Fig. 6. Optimal transmission range of an ad-hoc link changes with BS footprint and spectrum allocation. Note that $\delta=100 \mathrm{~m}$ and $S^{\prime}=400 \mathrm{~m}$.

Simulation settings: In the simulations, an ideal "reliable" media access protocol is assumed. A fair schedule (global knowledge is assumed) that provides the max-min fairness is implemented. Minimum-hop routing is enforced in the ad-hoc component (in line with the analysis). The results with random placements of users are averages from 20 simulations, each of which uses a randomly generated scenario and runs for 15 seconds.

In the hybrid network, the diameter of a cell is $2 \mathrm{~km}$ (i.e. $D=1000 \mathrm{~m})$. In the first set of experiments, there are 10 tiers of users (i.e., $n=10$ ) and the distance between adjacent users is $100 \mathrm{~m}$ (i.e., $\delta=100 \mathrm{~m}$ ). With these settings, the total number of users in a cell is 330 . For random placements of users, each of the 330 users is randomly positioned within the hexagonal cell. Note here that this number is reasonable, since the BS's footprint in todays cellular networks covers roughly $2 \mathrm{~km}$, and one might expect the number of users to be fairly large.

The size of the BS footprint ${ }^{9} b^{\prime}$, varies from $100 \mathrm{~m}$ to $1000 \mathrm{~m}$. The transmission range of an ad-hoc link $r^{\prime}$ varies from $100 \mathrm{~m}$ to $400 \mathrm{~m}$ and the sensing range $S^{\prime}$ is fixed at $400 \mathrm{~m}$ (see footnote 4). The total spectral bandwidth $B$ is $1.25 \mathrm{MHz}$ and the spectral allocations that we consider are $\gamma=0.1,0.3,0.5,0.7$ and 0.9 . The path-loss exponent $\alpha=4^{10}$.

Optimizing the transmission range of an ad-hoc link: An increase in the transmission range of an ad-hoc link reduces the total relay load in the ad-hoc component (see Equation (7)); however, it also reduces the transmission rate that can be sustained on each link (see Equation (9)). Striking the balance between the two is necessary for the highest capacity. To understand this effect, we compute the capacity of the hybrid network (using our analysis) by varying the transmission range of the ad-hoc links in an exhaustive set of scenarios; our goal is to determine the optimal transmission range in each of these scenarios. Fig. 6 depicts the transmission range that finds the highest capacity of the network. We observe that the optimal transmission range varies with the size of the BS footprint and the fraction of the spectrum allocated to the infrastructure component $(\gamma)$. First, note that the optimal transmission range decreases when $\gamma$ increases. When $\gamma$ is large, the portion of the spectrum that is allocated for ad-hoc transmissions is small. In this regime, in order to achieve a higher $\lambda_{A}$, a smaller transmission range should be used in order to increase the link rate of ad-hoc transmissions. Second, we observe that the

\footnotetext{
${ }^{9}$ Recall that in our framework, $b, r$ and $S$ are defined in terms of units of length $\delta$; we will use $b^{\prime}, r^{\prime}$ and $S^{\prime}$ to represent the actual values (computed with the corresponding value of $\delta$ ) of these variables.

${ }^{10}$ Without loss of generality, we use the same value of $\alpha$ for both the direct BS to user link and the intra-user links. It is easy to extend the results if the value is different in the two cases.
} 
optimal transmission range increases with the size of the BS footprint. When the size of the BS footprint increases, the bottleneck on the capacity of the hybrid network is the capacity of the infrastructure component since the BS now supports a large number of directly connected users, to which it has links with poor channel qualities. In this regime, choosing a larger transmission range for ad-hoc link transmissions will lead to a higher $\lambda_{I}$. This is because, with a larger transmission range, users at the boundary of the infrastructure and the adhoc components can reach proxies that are closer to the BS. These proxies have higher ILCs and this in turn, translates to a higher network capacity. This can be easily seen from the maximization problem defined in Equation 3.

Capacity of the hybrid network: For the hybrid network, the analytical results and the simulation results with both the regular and random placements of users are depicted in Fig. 7. In the figure, we see that the simulation results with regular placements of users exhibit similar behaviors and conform with the analytical results ${ }^{11}$. The slight discrepancies between the results are due to the approximation in the analysis that the ILCs of users that are located within the same tier are the same and equal to the best ILC possible for any user within the tier (See Section IV). Note that the results depicted in Fig. 7 are obtained by selecting the ad-hoc transmission range that maximizes the uniform throughput of the hybrid network. The uniform throughput of the corresponding pure cellular network is represented by the bold solid lines. The hybrid network yields capacity improvements over the corresponding pure cellular network in certain parametric regimes. However, due to the capacity bottleneck of the infrastructure component when $\gamma$ is small $(\gamma \leq 0.3)$, the uniform throughput of the hybrid network is poor in general. However, when $\gamma$ increases, the hybrid network begins to outperform the pure cellular network, as long as the BS footprint is not too large. When $\gamma$ increases, the size of the BS footprint for achieving the maximum uniform throughput also increases. This is because a higher $\gamma$ means a lower ad-hoc component capacity. In order for the ad-hoc component to achieve a high uniform throughput, the size of the BS footprint must be increased so that the relay load in the ad-hoc component decreases. Again, due to the two contradicting factors that are in play (discussed in the previous paragraph) "sweet spots" are observed with relatively large $\gamma$ values. Generally, the improvements with the hybrid network over the corresponding original pure cellular network can be as great as $70 \%$ when $\gamma, b$ and $r$ are appropriately selected. The capacity gains with the hybrid network are due to the better exploitation of spatial reuse in the ad-hoc component (more simultaneous ad-hoc transmissions can happen) and the increased bit rates due to the use of shorter range links.

Regular versus Random Deployment: In Fig. 7, we see that the simulation results with random placements of users conform with the simulation results with regular placements of users. We have observed that the slight discrepancies are due to an increase in the relay overhead which is caused by an increase in the average length of the relay paths in the random scenarios. In particular, we observe an increase, on

\footnotetext{
${ }^{11}$ When $r^{\prime}=100 \mathrm{~m}$, some of the users are disconnected in the random scenarios due to the short transmission range of an ad-hoc link. In this case, the uniform throughput of the hybrid network is thus equal to zero as per our definition.
}
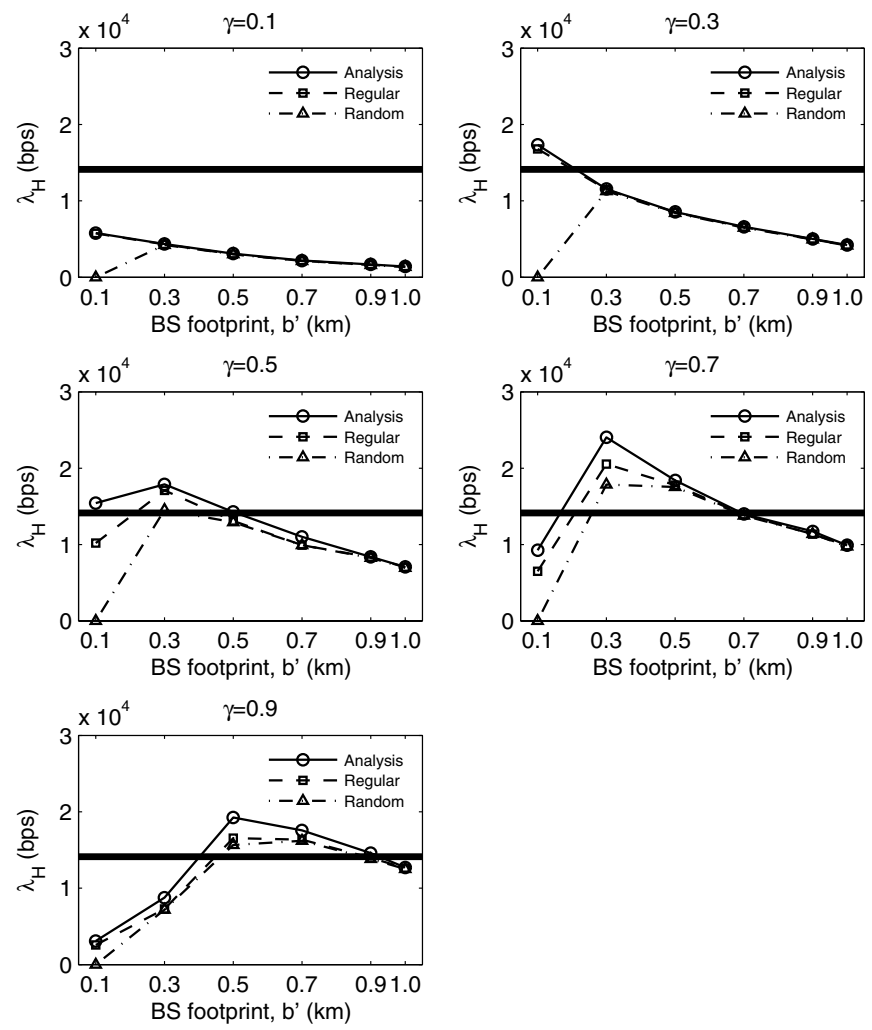

Fig. 7. The uniform throughput of the hybrid network with different sizes of BS footprint $\left(b^{\prime}\right)$ and spectrum allocations $(\gamma)$. An optimal transmission range of an ad-hoc link is used in each of the scenarios. The solid line represents the capacity of the corresponding cellular network.

average, in the total relay load from $0.6 \%$ to $16 \%$ in the random scenarios. This results in a slight degradation in capacity as seen in Fig. 7. However, due to the uniform distribution of users, the topological properties of the network with random placements are fairly similar to that with regular placements of users [26]. In the case of random deployments however, if the transmission range of a user is too short relative to the interuser distance, the connectivity of the users to the proxies will degrade. In other words, one must ensure that the transmission range of a user is long enough to ensure that all users can reach the proxies with high probability. In our framework, the transmission range of a user is $r \delta$ and the inter-user distance is $\delta$. With regular placements, it suffices to have $r=1$ in order to ensure complete connectivity. We observe that with the random deployment a value of $r=2$ is enough to provide complete connectivity with a probability $\approx 1$.

Impact of density of users in the network: Next, we investigate the effects of varying the user density on the capacity of the hybrid network. In Fig. 8(a), we depict the maximum capacity improvements of the hybrid network over the corresponding pure cellular network with different user densities (varied by adjusting the number of tiers of users in a cell). When the user density is low, the capacity improvements are also low. In this regime, since adjacent users are separated by large distances, a large transmission range has to be used on the ad hoc links in order to maintain connectivity. This in turn limits the achievable transmission rates on the ad-hoc links and thus, reduces the capacity. On the other hand, as the user density increases, the capacity improvements become better. As 


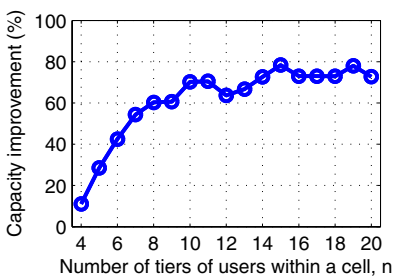
$=1000 \mathrm{~m}$. (a) Varying users density. Cell radius

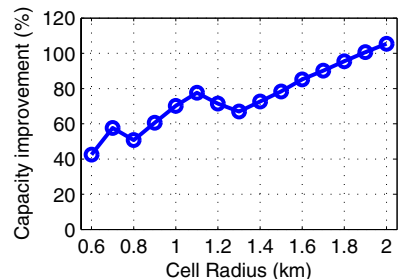

(b) Varying cell size. User density is fixed $(\delta=100 \mathrm{~m})$.
Fig. 8. The maximum capacity improvements provided by the hybrid network over the corresponding pure cellular network with (a) different density of users and (b) different cell sizes.

the user density increases, the distance between adjacent users decreases and this leads to two advantages: first, one can now establish shorter links and thereby, higher transmission rates can be achieved. Second, it improves the flexibility/granularity of the transmission range selection. While the benefit provided by the second advantage is not immediately apparent, it is vital for maximizing the uniform throughput in the ad-hoc component (note as discussed earlier, the capacity varies with $r$ ). Note that after a certain density, the capacity improvements converge to a stable saturated value $(\approx 70 \%)$. This is because the rates cannot be increased beyond a certain level (as dictated by the Shannon Capacity and the available raw bandwidth).

Impact of cell size: We evaluate the impact of the cell size on the maximum capacity improvements that can be achieved with the hybrid network. In Fig. 8(b), we depict the results by varying the cell radius. Note that the user density is fixed $(\delta=100 \mathrm{~m})$. Therefore, when the size of the cell increases, the number of tiers of users in a cell increases. From the results, we see that the capacity improvements generally increase with an increase in the cell size. This seems counter-intuitive at first; however, note that the capacity of the corresponding cellular network decreases when the cell size increases since there are more users with poor channel qualities. With the hybrid network these users benefit to a greater extent from the highrate ad hoc transmissions. It may seem that the penalty due to multihop transmissions will increase with cell size could hurt capacity; however, since the sensing range is fixed, increasing the cell size can actually increase the number of simultaneous ad hoc transmissions in the network. The combined effects result in a slight increase in the capacity of the hybrid network when the cell size increases.

\section{Conclusions}

In this paper, we consider an approach of augmenting cellular networks with ad-hoc wireless connectivity to improve spatial reuse. In particular, we reduce the coverage area of the base station and require that users outside the area, rely on other users within the area for connectivity. Our objective is to evaluate if this approach can yield capacity benefits over that of the original cellular network. While the use of the relays to form a hybrid network provides shorter higher rate communication links, multihop forwarding contributes to a reduction in capacity. We analytically compute the capacity of a regular hexagonal hybrid network under the conditions of max-min fairness. We also perform simulations with both deterministic and random placements of users. We observe that the hexagonal hybrid network is able to provide higher capacities than a corresponding pure cellular network. With careful parametric choices, the capacity of the hexagonal hybrid network can exceed that of the corresponding pure cellular network by as much as $70 \%$.

\section{ACKNOWLEDGMENTS}

The authors wish to thank Matt Grob, Rajesh Pankaj, David Schultz, Vikram Gupta and other QUALCOMM staff for suggestions and comments.

\section{REFERENCES}

[1] B. Liu, Z. Liu, and D. Towsley, "On the Capacity of Hybrid Wireless Networks," in IEEE INFOCOM, 2003, pp. 1543-1552.

[2] H. Luo, R. Ramjee, P. Sinha, L. E. Li, and S. Lu, "UCAN: A Unified Cellular and Ad-Hoc Network Architecture," in ACM MOBICOM, 2003.

[3] Y.-D. Lin and Y.-C. Hsu, "Multihop Cellular: A New Architecture for Wireless Communications," in IEEE INFOCOM, 2000.

[4] H. Wu, C. Qiao, S. De, and O. Tonguz, "Integrated Cellular and Ad Hoc Relaying Systems: iCAR," IEEE JSAC, vol. 19, no. 10, 2001.

[5] H.-Y. Hsieh and R. Sivakumar, "On Using the Ad-hoc Network Model in Cellular Packet Data Networks," in ACM MOBIHOC, 2002.

[6] R. Chakravorty, S. Agarwal, S. Banerjee, and I. Pratt, "MoB: A Mobile Bazaar for Wide-area Wireless Services," in ACM MOBICOM, 2005.

[7] H. Viswanathan and S. Mukherjee, "Performance of Cellular Networks with Relays and Centralized Scheduling," in IEEE VTC, 2003.

[8] A. Agarwal and P. R. Kumar, "Capacity Bounds for Ad hoc and Hybrid Wireless Networks," ACM SIGCOMM CCR, vol. 34, no. 3, 2004.

[9] S. Toumpis, "Capacity Bounds for Three Classes of Wireless Networks: Asymmetric, Cluster, and Hybrid," in ACM MOBIHOC, 2004.

[10] J. Cho and Z. J. Haas, "On the Throughput Enhancement of the Downstream Channel in Cellular Radio Networks Through Multihop Relaying," IEEE JSAC, vol. 22, no. 7, 2004.

[11] S. Mukherjee and H. Viswanathan, "Analysis of Throughput Gains from Relays in Cellular Networks," in GLOBECOM, 2003.

[12] H. Zhai and Y. Fang, "Physical Carrier Sensing and Spatial Reuse in Multirate and Multihop Wireless Ad Hoc Networks," in IEEE INFOCOM, 2006.

[13] S. Sarkar and K. N. Sivarajan, "Fairness in Cellular Mobile Networks," IEEE Transactions on Information Theory, vol. 48, 2002.

[14] A. Balachandran, G. Voelker, P. Bahl, and P. V. Rangan, "Characterizing User Behavior and Network Performance in a Public Wireless LAN," in ACM SIGMETRICS, 2002.

[15] P. Gupta and P. R. Kumar, "The Capacity of Wireless Networks," IEEE Transactions on Information Theory, vol. 46, pp. 388-404, 2000.

[16] T. Bonald and A. Proutiere, "Wireless Downlink Data Channels: User Performance and Cell Dimensioning," in ACM MOBICOM, 2003.

[17] X. Yang and N. H. Vaidya, "On Physical Carrier Sensing in Wireless Ad Hoc Networks," in IEEE INFOCOM, 2005.

[18] C. E. Shannon, "Communication in the presence of noise," in IRE, 1949, pp. 10-21.

[19] A. Bedekar, S. Borst, K. Ramanan, and P. Whiting, "Downlink Scheduling in CDMA DATA Networks," in GLOBECOM, 1999.

[20] P. Bender, P. Black, M. Grob, R. Padovani, N. Sindhushayana, and A. Viterbi, "CDMA/HDR: A Bandwidth-Efficient High-Speed Wireless Data Service for Nomadic Users," IEEE Communications Magazine, 2000.

[21] S. Lu, V. Bharghavan, and R. Srikant, "Fair Scheduling in Wireless Packet Networks," IEEE Transactions on Networking, vol. 7, 1999.

[22] T.-S. Kim, H. Lim, and J. C. Hou, "Improving Spatial Reuse through Tuning Transmit Power, Carrier Sense Threshold, and Data Rate in Multihop Wireless Networks," in ACM MOBICOM, 2006.

[23] J. Li, C. Blake, D. S. J. D. Couto, H. I. Lee, and R. Morris, "Capacity of Ad Hoc Wireless Networks," in ACM MOBICOM, 2001, pp. 61-69.

[24] P. Santi, Topology control in Wireless Ad hoc and Sensor Networks. John Wiley \& Sons, 2005

[25] S. McCanne and S. Floyd, "Ns-2 simulator," http://www.isi.edu/nsbam/ns/.

[26] J. Robinson and E. Knightly, "A Performance Study of Deployment Factors in Wireless Mesh Networks," in IEEE INFOCOM, 2007. 